\title{
Wiedergutmachung - Strafmilderung nur nach Geständnis
}

\author{
BGH - 1 StR 405/02 (LG Konstanz) = StraFo 2003, \\ S. $248-251$; NJW 2003, S. 1466 - 1469.
}

Jan Markus Schulte

Sachverhalt (gekürzt):

$\mathrm{N}$ achdem sie sich in einer Diskothek kennengelernt hatten, gingen der Angeklagte und die 20-jährige Geschädigte zu einer nahegelegenen Autowaschanlage. Dort hielt er sie fest, drückte sie gegen eine Wand und nahm gegen ihren Willen sexuelle Handlungen an ihr vor. Er versuchte, mit seinem Penis in ihre Scheide einzudringen und führte an ihr den Oralverkehr aus. Dabei fügte er dem Opfer Kratzwunden sowie erhebliche Schmerzen zu.

In der Hauptverhandlung zielte die Verteidigung des Angeklagten auf Freispruch ab; es wurde unter Beweis gestellt, die Angeklagte würde sich vor der Diskothek prostituieren und ein Sachverständigengutachten zum Beweise, die Geschädigte habe die Unwahrheit gesagt, beantragt. Vor Beginn der Hauptverhandlung und nach der Vernehmung des Tatopfers wies die Kammer gemäß $\S 155 \mathrm{a}$ StPO auf die Möglichkeit eines Ausgleichs hin.

Daraufhin räumte der Angeklagte ein »Mißverständnis bzw. ein Verschulden « ein, entschuldigte sich -nach Auffassung der Kammer ernsthaftbeim Tatopfer, bot an, sich durch Vermittlung eines Sozialtherapeuten mit dem Tatopfer an einen Tisch zu setzen und ihr durch ein Gespräch dabei zu helfen, »die Sache endgültig zu verarbeiten«. Er erklärte sich zudem bereit, zum Ausgleich des immateriellen Schadens ein Schmerzensgeld von 3.500,- Euro zu bezahlen. Der Vater des Angeklagten übergab nach den Schlußvorträgen -der Verteidiger hatte zuvor Freispruch beantragt- einen von mehreren ebenfalls anwesenden Familienmitgliedern gesammelten Betrag von 2.500 Euro der Nebenklage und sagte zu, im Laufe des Tages weitere 1.000 Euro beizubringen. Die Geschädigte nahm diesen Betrag »durch ihren Beistand als gewissen Ausgleich « entgegen.
Leitsatz:

Bei Gewaltdelikten und Delikten gegen die sexuelle Selbstbestimmung ist für einen erfolgreichen Täter-Opfer-Ausgleich mit der zu Gunsten des Angeklagten wirkenden Folge der Strafmilderung nach $\S 49$ Abs. 1 StGB regelmäßig ein Geständnis zu verlangen.

\section{Anmerkung:}

Revisionsentscheidungen zum Täter-Opfer-Ausgleich sollten angesichts der Zielrichtung dieses Instruments zur informellen konsensualen Verfahrenserledigung leichter bis mittlerer Kriminalität Seltenheitswert haben. Aber dies ist nur die halbe Wahrheit: Wiedergutmachung ist auch bei schweren Delikten ein gesetzlicher Strafmilderungsgrund und somit ein Anreiz für die Strafverteidigung, ein versöhntes Opfer zu präsentieren. Die wachsende Bedeutung der Wiedergutmachung ist Ergebnis einer stillen Reform, die Opfern eine aktivere Rolle im Strafverfahren zumißt und Beschuldigten einen Anreiz schafft, sich mit dem Opfer zu einigen bzw. Wiedergutmachungsleistungen zumindest anzubieten.

Möglich sind solche Absprachen in allen Verfahrensstadien, insbesondere schon im für die Praxis wichtigsten Bereich, der Einstellung des Verfahrens nach Ende der Ermittlungen. Hingegen ist es für die Klärung des Strafmilderungsgrundes nötig, die eher vagen und weiten Merkmale des insoweit zentralen $\S 46 a$ StGB zu präzisieren. Leider hat die Rechtsprechung für den schon seit neun Jahren geltenden $\S 46 a$ StGB noch keine klare Linie gefunden ${ }^{1}$. Nur eine Regel ist sicher: wenn ein Sachverhalt Anzeichen für einen Ausgleich bietet, muss das Gericht die Anwendung des $§ 46 \mathrm{a}$ StGB stets in den Urteilsgründen erörtern ${ }^{2}$.

Im Rahmen des $\S 46 a$ StGB unterscheidet der Gesetzeswortlaut zwei Varianten:
- Nr. 1 verlangt ein ernsthaftes Erstreben oder mindestens Bemühen der Tatwiedergutmachung, während

- Nr. 2 eine überwiegende Schadenswiedergutmachung unter erheblichen persönlichen Leistungen oder Verzicht vom Täter verlangt.

Interpretierte der BGH zunächst den Gesetzeswortlaut dahingehend, als behandele Nr. 1 den immateriellen Schaden und sei damit hauptsächlich auf Nichtvermögensdelikte anwendbar, während Nr. 2 den materiellen Schadensersatz, d.h. insbesondere Vermögensdelikte betreffe ${ }^{3}$, geht die Rechtswissenschaft ${ }^{4}$ - neuerdings auch für den BGH überzeugend ${ }^{5}$ - davon aus, in beiden Varianten des § 46a StGB unterschiedliche Leistungsarten zu sehen. Danach stehen bei $\S 46 \mathrm{a}$ StGB einmal die immateriellen Leistungen $(\S 46 \mathrm{a}$ Nr. 1 StGB), einmal die materiellen Leistungen (§ 46a Nr. 2 StGB) durch den Täter im Vordergrund, es kann jedoch nie auf eine Leistungsart völlig verzichtet werden ${ }^{6}$.

Jedoch kommt es unabhängig von der Frage, ob die Nr. 1 und Nr. 2 des $\S 46 a$ StGB nun unterschiedliche Leistungs- oder Schadensarten beschreiben, für beide Auslegungen darauf an, wie die immaterielle Ausgleichskomponenten zu bestimmen sind, wann vom Vorliegen der »weichen« Merkmale des $\S 46 a$ StGB, also einem ernsthaften Bemühen des Täters um Ausgleich oder seinem erheblichem Verzicht bei der Schadenswiedergutmachung zu Gunsten des Opfers, gesprochen werden kann.

Die Rechtsprechung des BGH konnte bisher nicht viel zu einer Klärung dieser Frage beitragen. Zwar wurde das Erfordernis eines »kommunikativen Kontakts « zwischen Täter und Opfer aufgestellt ${ }^{7}$, doch umschreibt dieses Merkmal wiederum nur, daß es für den »Ausgleich « nach $\S 46 a$ Nr. 1 StGB zwei Seiten geben muß, die sich verständigen, die also in einen »kommunikativen Kontakt « zueinander treten. Über den besonderen Inhalt dieses Kontakts herrscht - je- 
denfalls was die Rechtsprechung der Revisionsgerichte betrifft - nach wie vor Unklarheit. Wurde etwa vom zweiten Strafsenat ein Geständnis des Täters bei einer Körperverletzung für den Ausgleich nicht zwingend gefordert ${ }^{8}$, sieht das vorliegende Urteil des ersten Strafsenats nur drei Monate später vor, dass jedenfalls bei Gewaltdelikten und Delikten gegen die sexuelle Selbstbestimmung ein Geständnis regelmäßig zu verlangen $\operatorname{sei}^{9}$.

Hatte die Rechtsprechung die Vorschrift des $\S$ 46a StGB zunächst sehr weit ausgelegt ${ }^{10}$, so zeigen gerade einige jüngere Entscheidungen einen spürbaren Unwillen, insbesondere Tätern von Sexualdelikten die Vergünstigung des $\S 46 \mathrm{a}$ StGB so ohne weiteres zuzusprechen: es wurden "grundlegende Bedenken gegenüber der gesetzlichen Ausgestaltung der Vorschrift des $\S 46 a \mathrm{StGB}$ « geäußert, der BGH fühlte sich zur Aufhebung von $\mathrm{zu}$ großzügigen Urteilen "genötigt « ${ }^{11}$. So wurde bei Sexualdelikten trotz Schmerzensgeldzahlungen des Täters eine Schadenswiedergutmachung nach $\S 46 a$ Nr. 2 StGB noch nicht einmal in Betracht gezogen ${ }^{12}$ und zur Versagung des Anwendungsbereichs des $§ 46 \mathrm{a}$ Nr. 1 StGB dem Opfer unterstellt, es hätte entsprechende Zahlungen des Täters nur aus der Not heraus akzeptiert, damit entfalle aber die friedensstiftende Wirkung des Ausgleichs; zugleich sei kein Raum für ein »Bemühen « des Täters um einen Ausgleich, wenn diesem die finanzielle Notlage des Opfers bekannt sei ${ }^{13}$.

Gerade gegen die letztgenannte Entscheidung ist eingewandt worden, daß sich der mitgeteilte Sachverhalt auch anders interpretieren ließe, eine innere Akzeptanz des Ausgleichs durch das Opfer nicht abwegig sei, da in einer »Täter-Opfer-Aus-

Wiedergutmachung ist auch bei Verbrechen ein gesetzlicher Strafmilderungsgrund und damit für den geständigen Beschuldigten ein Anreiz für Angebote an das Opfer

gleich « betitelten anwaltlichen Vereinbarung das Opfer erklärte, kein Interesse mehr an einer Bestrafung des Täters zu haben und entsprechend seine Nebenklage zurückzog ${ }^{14}$. Dies könnte um so mehr überzeugen, da der erste BGH-Senat selbst die Rücknahme der Nebenklage als Zeichen für eine Akzeptanz des Ausgleichs angesehen hat ${ }^{15}$ freilich betraf diese Entscheidung gerade kein Sexualdelikt sondern eine Körperverletzung.

Anders als Schmerzensgeldzahlungen sind Schadensersatzzahlungen gemeinhin leicht $\mathrm{zu}$ bestimmen. Zivilrechtlich ist der Täter zu deren (vollen) Leistung verpflichtet ${ }^{16}$, doch wurde auch hier für eine Versagung der Strafmilderung bei einem erst sehr spät erfolgenden materiellen Aus-
Dagegen versucht die Gesetzgebung, die sich zunächst nicht auf die Formalisierung eines Ausgleichsverfahrens bei $\S 46 a$ StGB festlegte, nun die Vollstreckbarkeit zivilrechtlicher Ansprüche eines Opfers im Strafverfahren $\mathrm{zu}$ verbessern, entweder im Hauptverfahren ${ }^{20}$ oder sogar schon vor dessen Eröffnung21. Dies macht deutlich, dass es anerkannter Kriterien für einen immateriellen Aus-

gleichs auf die Schwere des zugrundeliegenden

Summarisch betrachtet läßt sich festhalten, devisionsgerichte je nach Schwere des forderungen stellen. Vordergründig könnte dies dem Willen des Gesetzgebers, der mit der Fassung des § 46a StGB ja gerade eine Vielzahl von der Instanzgerichte erfassen wollte, widersprechen. Wenn aber die Hürden zur Erfüllung des § wieso dann sogar bei einem Verbrechen eine Strafminderung zulässig sein soll bzw. ein Straf-

Dogmatisch wird dies damit begründet, die verhalten, werden könne, wenn es ein gewisses Gewicht aufweise ${ }^{18}$. Danach ist es nicht entscheidend, welche Anforderungen an einen Ausgleich getungen des Täters in Anbetracht des konkret verschuldeten Unrechts erscheinen. Ausgleich hinzunehmender Tribut an die Kontrollfunktion der Revisionsgerichte. Aber dort hält sich erprüfbarkeit der Anwendung fakultatiGrenzen; letztlich reagieren sie nur, wenn die Strafzumessung des Instanzgerichts $\mathrm{zu}$ seh nach einem »Freikauf « riecht. Zu einer überzeugenden Dogmatik des $\S 46$ a StGB kann dies nicht führen. gleich bedarf, da in weit größerem Maße als bis-
her $^{22}$ zivilrechtliche Opferansprüche und damit die materiellen Komponenten eines Ausgleichs schon im Strafverfahren erledigt werden könnten.

Erfolgt die Beendigung eines Verfahrens im Einverständnis aller Beteiligten, kann dem Genugtuungsinteresse des Opfers hierbei in erster Linie mit der Sicherung der Vollstreckbarkeit zivilrechtlicher Schadensersatz- und Schmerzensgeldansprüche im Rahmen der richterlichen $\mathrm{Zu}$ stimmung zur Verfahrenseinstellung gedient werden. Dabei kann die Zustimmung des Täters als genügende immaterielle Leistung für $\S 46 \mathrm{a}$ Nr. 2 StGB betrachtet werden, es kommt darauf $\mathrm{zu}$ einer Schadensregulierung allein noch nicht an, daß die Befriedigung der Opferansprüche für ihn eine Last im Sinne erheblicher persönlicher Leistungen oder Verzicht ist. Allerdings ist auch die Durchsetzbarkeit zivilrechtlicher Abwehransprüche mit Verhaltensauflagen an den Täter etwa aus $\S 1004$ BGB denkbar, durch die das ernsthaftes Erstreben einer Wiedergutmachung demonstriert werden könnte.

Unabhängig von den Detailfragen ist die eigentliche Bedeutung einverständlicher Verfahrenserledigungen jedoch in der Auflösung der traditionellen Trennung von Straf- und Zivilrecht zu sehen, ein Sachverhalt wird nur noch einmal vor den Richter gebracht, dies ist ja nicht nur prozeßökonomisch einleuchtend.

\section{Anmerkungen:}

1 Einen Überblick zur Rechtsprechung bietet Franke, NStZ 2003, S. 410ff; kritisch: Oberlies, Streit 2000, S. 99ff.

2 BGH, StV 2000, S. 129; OLG Hamm, StV 1999, S. 89,90 .

3 BGH, StV 1995, S. 464, 465; Tröndle-Fischer, Strafgesetzbuch und Nebengesetze, 51. Aufl. § 46a Rn. 10, 11.

4 Lackner / Kühl, Strafgesetzbuch mit Erläuterungen, 24. Aufl. § 46a Rn. 2; Schöch, TäterOpfer-Ausgleich und Schadenswiedergutmachung gemäß § 46a StGB, in: 50 Jahre BGH Festgabe aus der Wissenschaft, S. 309ff.

5 BGH, StraFo 2001, S. 425 .

6 Schöch (o. Fn. 4), S. 336.

7 der nicht zwingend über professionelle Dritte oder den Verteidiger des Angeklagten erfolgen muß.

8 BGH, StV 2002, S. 649.

9 BGH, StraFo 2003, S. 248 mit krit. Anm. Götting.

10 BGH, StV 1995, S. 249; BGH, NStZ-RR 1998, S. 297; LG Potsdam NJ 1998, S. 214

11 BGH, StV 2000, S. 129

12 BGH, NStZ-RR 2002, S. 263 und BGH, NStZ-RR, 2002, S. 329.

13 BGH, NJW 2002, S. 3264.

14 Götting (o. Fn. 9), S. 252.

15 BGH, NStZ-RR 1998, S. 297.

16 eine vollständige Entschädigung des Opfers ist nicht zwingende Voraussetzung des § 46a StGB.

17 OLG Stuttgart, NJW 1996, S. 2109; BGH, StV 1995, S. 249

18 Stein, NStZ 2000, S. 396.

19 so der Titel eines Beitrags von Kilching, TOAInfodienst Nr. 12, 2000, S. $26 \mathrm{ff}$.

20 durch den Entwurf eines OpferrechtsreformG. Dieser sieht u.a. ein reformiertes Adhäsionsverfahren vor; vgl. den Entwurf der CDU/CSU, BTDs. $15 / 814$.

21 so der Entwurf der Bundesregierung, Pressemitteilung Nr. 90/03 vom 05.11.03.

22 ein Versuch einer Bestandsaufnahme der Anwendung des § 46a StGB bietet Puderbach, Täter-Opfer-Ausgleich im Ermittlungs- und Hauptverfahren, in: Egg, Minthe (Hrsg.), Opfer von Straftaten, S. $97 \mathrm{ff}$.

Jan Markus Schulte ist wissenschaftlicher Mitarbeiter am Kriminologischen Institut der CAU Kiel 